\title{
Ion formation upon electron collisions with valine embedded in helium nanodroplets ${ }^{\star}$
}

\author{
Nikolaus Weinberger ${ }^{1}$, Stefan Ralser ${ }^{1}$, Michael Renzler ${ }^{1}$, Martina Harnisch ${ }^{1}$, Alexander Kaiser ${ }^{1}$, Stefan Denifl ${ }^{1}$, \\ Diethard K. Böhme ${ }^{2}$, and Paul Scheier ${ }^{1, a}$ \\ 1 Institut für Ionenphysik und Angewandte Physik, Universität Innsbruck, Technikerstr. 25, A-6020 Innsbruck, Austria \\ 2 Department of Chemistry, York University, 4700 Keele St., Toronto, ON, Canada M3J 1P3
}

Received 30 December 2015 / Received in final form 7 March 2016

Published online 19 April 2016

(C) The Author(s) 2016. This article is published with open access at Springerlink.com

\begin{abstract}
We report here experimental results for the electron ionization of large superfluid helium nanodroplets with sizes of about $10^{5}$ atoms that are doped with valine and clusters of valine. Spectra of both cations and anions were monitored with high-resolution time-of-flight mass spectrometry (mass resolution $>4000$ ). Clear series of peaks with valine cluster sizes up to at least 40 and spaced by the mass of a valine molecule are visible in both the cation and anion spectra. Ion efficiency curves are presented for selected cations and anions at electron energies up to about $40 \mathrm{eV}$ and these provide insight into the mode of ion formation. The measured onset of $24.59 \mathrm{eV}$ for cations is indicative of valine ionization by $\mathrm{He}^{+}$whereas broad resonances at 2, 10 and $22 \mathrm{eV}$ (and beyond) in the formation of anions speak to the occurrence of various modes of dissociative electron attachment by collisions with electrons or $\mathrm{He}^{*-}$ and the influence of droplet size on the relative importance of these processes. Comparisons are also made with gas phase results and these provide insight into a matrix effect within the superfluid helium nanodroplet.
\end{abstract}

\section{Introduction}

In recent years interest in collisions of leptons, photons or heavier species with biomolecules has increased substantially [1-6] as the description of radiation damage of biological tissue remains incomplete at the molecular level. Apparently secondary species formed by high-energy radiation, such as electrons, radicals and ions, are responsible for the chemical transformation of biomolecules that accompanies severe radiation damage in cells, viz. biomolecules such as amino acids, the building blocks of proteins. Amino acids are characterized by two functional groups, the amine $\left(-\mathrm{NH}_{2}\right)$ and the carboxyl $(-\mathrm{COOH})$, and possess a side-chain specific for each amino acid compound. Here we investigate electron interactions with clusters of the amino acid valine $\left(\left(\mathrm{CH}_{3}\right)_{2} \mathrm{CHCH}\left(\mathrm{NH}_{2}\right) \mathrm{COOH}\right)$ embedded in helium droplets. Valine is important in the biosynthesis of proteins in humans who acquire valine through their diet. Previous gas-phase studies with isolated valine have provided valuable insight into the stability of this molecule against dissociation by particle collisions [7-15]. Low-energy electron interactions with

\footnotetext{
* Contribution to the Topical Issue "Advances in Positron and Electron Scattering", edited by Paulo Limao-Vieira, Gustavo Garcia, E. Krishnakumar, James Sullivan, Hajime Tanuma and Zoran Petrovic.

a e-mail: paul.scheier@uibk.ac.at
}

isolated valine turn out to be particularly dissociative. In cation formation at an electron energy of about $70 \mathrm{eV}$ (close to the maximum of the ionization cross section) the molecular ion intensity is less than $1 \%$ of the intensity of the most abundant fragment ion formed by the loss of the neutral carboxyl group [10]. In the case of resonant anion formation the un-fragmented molecular anion cannot be observed at all $[9,14,15]$. Instead, the dehydrogenated parent anion is formed as the most abundant anion with the main resonance at the electron energy of $1.2 \mathrm{eV}$ and other fragment anions can be observed at higher electron energies. The dynamics of the dissociation process also has been monitored by investigating metastable decays in the $\mu$ s time regime and in this way it was shown that, at $5.3 \mathrm{eV}$, the dehydrogenated parent anion, formed as an intermediate, further decays into $\mathrm{COOH}^{-}$and neutral fragment(s) [15].

The behavior of fragmentation induced by energetic particles may change substantially from isolated molecules to molecular clusters that represent the condensed phase [16-18]. The excess energy deposited by the projectile in the presence of other molecules or a solvent may be transferred to the surrounding species and lead to buffering effects in dissociation. Cluster-specific effects include, for example, novel fragmentation channels [18] or intramolecular bond-weakening due to the intermolecular bonds in the cluster [16]. Clustering occurs in our 
experiments when valine molecules are embedded in superfluid helium nanodroplets at the extremely low temperature of $0.37 \mathrm{~K}$ [19]. Few studies on biomolecules embedded in helium nanodroplets have been carried out so far [20-26]. Noteworthy is a successful experiment in which a protein ion with a mass of $12000 \mathrm{u}$ was embedded in a droplet [27]. Most electron collision experiments in helium droplets have involved mixed clusters with other amino acids as well as with water [20-23].

Our group has previously studied the electron ionization of helium droplets doped with valine using a sector field mass spectrometer (mass resolution $\sim 600$ ) and mean helium droplet sizes of about $10^{4}$ atoms [20]. Here we used a more powerful cryostat in the droplet source that allowed the generation of larger droplets with sizes of about $10^{5}$ atoms to accommodate larger dopant clusters. We employed high-resolution time-of-flight mass spectrometry (mass resolution $>4000$ ) to study in detail the cation and anion formation induced by electron collisions with valine embedded in large helium nanodroplets.

\section{Experimental set-up}

He nanodroplets (HNDs) were formed by expanding helium (purity 99.9999\%) from a stagnation pressure of about $2.3 \mathrm{MPa}$ through a $5 \mu \mathrm{m}$ nozzle, cooled by a closed-cycle refrigerator (Sumitomo Heavy Industries LTD, model RDK-415D) to about $9.7 \mathrm{~K}$, into vacuum. The size distribution of the neutral droplets was determined with a similar setup utilized previously [28]. From the log-normal size distribution we determined an average size of $1.5 \times 10^{5} \mathrm{He}$ atoms per droplet. This value agrees very well with the average droplet sizes reported in the literature $[19,29,30]$. The resulting supersonic beam was skimmed by a $0.8 \mathrm{~mm}$ conical skimmer, located $1 \mathrm{~cm}$ downstream from the nozzle and flew through two $10 \mathrm{~cm}$ long, differentially pumped pick-up regions. L-valine (grade BioUltra, $\geqslant 99.5 \%$ (NT), Sigma-Aldrich) was vaporized in an ohmically heated oven at a temperature of $340 \mathrm{~K}$ and picked up by the He droplets with almost unit efficiency. After the pickup region, the doped helium droplets passed a region in which they were ionized by collisions with electrons at energies ranging from 0 to $100 \mathrm{eV}$. Further details are specified in the figure captions. The ions were accelerated to $40 \mathrm{eV}$ into the extraction region of a commercial orthogonal time-of-flight mass spectrometer equipped with a reflectron (Tofwerk AG, model HTOF). For anions the mass resolution at the valine dimer was $m / \Delta m=4000$ and for cations 5000. The base pressure in the mass spectrometer was $10^{-5} \mathrm{~Pa}$. The ions were detected by a micro-channel plate and recorded via a time to digital converter. Additional experimental details have been described elsewhere [31,32]. Special homebuilt software was utilized to deduce ion intensities from the mass spectra taking into account all possible isotopologues [33]. In the latest version, this software can also extract ion efficiency curves of any ion of interest from a hdf5 file containing mass spectra as a function of the electron energy.

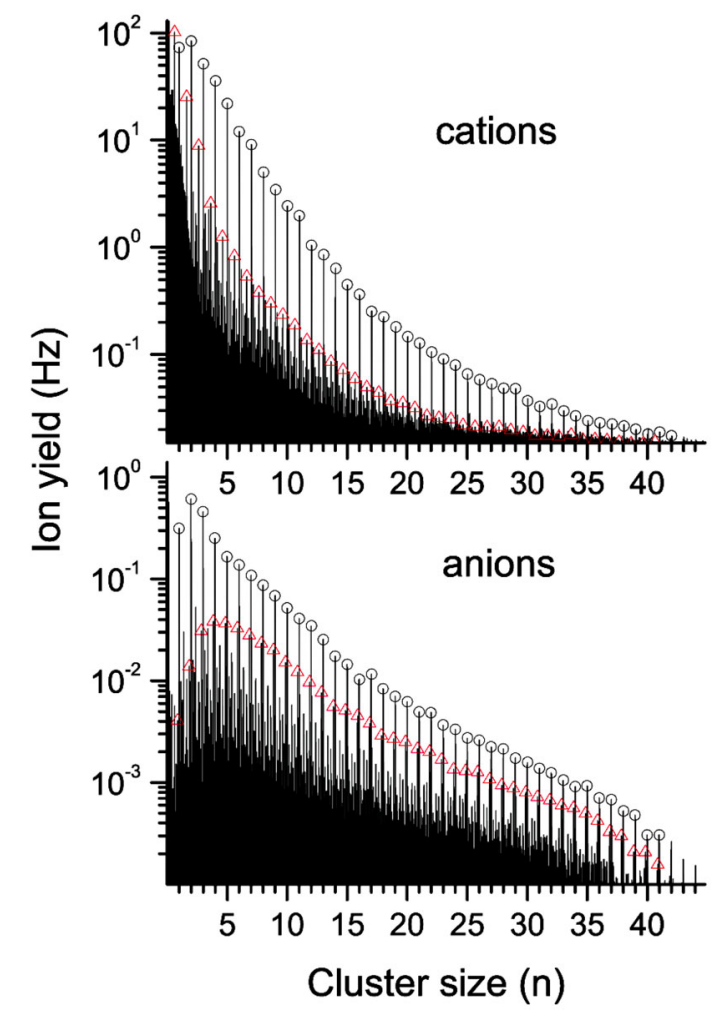

Fig. 1. Mass spectra for cations (top) and anions (bottom) formed upon electron irradiation of He nanodroplets doped with valine at $95 \mathrm{eV}(25 \mu \mathrm{A})$ and $22 \mathrm{eV}(64 \mu \mathrm{A})$, respectively. In the cation spectrum the circles represent the most abundant product ions $\mathrm{Val}_{n} \mathrm{H}^{+}$and the triangles represent the most abundant fragments $\left(\mathrm{Val}_{n}-\mathrm{COOH}\right)^{+}$. In the anion spectrum the circles indicate the maximum yield of $\left[\mathrm{Val}_{n}-\mathrm{H}\right]^{-}$and the triangles the position of the most abundant fragment $\left[\mathrm{Val}_{n}{ }^{-}\right.$ $\mathrm{OH}]^{-}$. Experimental conditions: pressure and temperature of the He before expansion: $2.3 \mathrm{MPa}$ and $9.75 \mathrm{~K}$ (average droplet size $1.5 \times 10^{5}$ ), temperature of the valine oven: $340 \mathrm{~K}$.

\section{Results and discussion}

\subsection{Mass spectra upon electron irradiation of valine embedded in He nanodroplets}

Figure 1 shows mass spectra for the positive and negative ions formed upon electron bombardment of He nanodroplets doped with valine at electron energies of 95 and $22 \mathrm{eV}$, respectively. Clear series of peaks spaced by the mass of a valine molecule are visible in both spectra with protonated parent cations $\mathrm{Val}_{n} \mathrm{H}^{+}$and the closedshell, dehydrogenated parent anions $\left[\mathrm{Val}_{n}-\mathrm{H}\right]^{-}$being the most abundant (except in the monomer region of the anions) with $n$ up to 40 . In both cases a repeating pattern of fragment ions is observed between these ions. In order to deduce the correct yield of all cations and anions contributing with their isotopic patterns to the measured mass spectrum we utilized our recently developed software isotope-fit [33]. There is perhaps a hint of weak intensity anomalies (magic numbers [34]) at $n=3,8,12,17,22$ and 27 , that indicate a special stability for these ions. 
Perhaps these ions have structures in which one or more valine complexes containing five valine molecules are attached to an ionic core of either $\mathrm{Val}_{2} \mathrm{H}^{+} /\left[\mathrm{Val}_{3}-\mathrm{H}\right]^{-}$or $\mathrm{Val}_{11} \mathrm{H}^{+} /\left[\mathrm{Val}_{12}-\mathrm{H}\right]^{-}$for cations and anions, respectively. As the pickup process should not favor particular cluster sizes and the binding energy that is released by the clustering of dopant molecules will quickly dissipate to the superfluid He matrix, we expect that the cluster size distribution of any dopant will not have any intensity anomalies before the ionization process. Evaporation of dopant molecules after the ionization process is required for the observation of magic numbers.

Both the parent cluster ions $\mathrm{Val}_{n}^{+}$and a number of product cluster cations were observed in the cation spectrum. The most abundant product cations were: $(\mathrm{Val})_{n-1} \mathrm{H}^{+}(85.1 \%),\left[\mathrm{Val}_{n}-\mathrm{COOH}\right]^{+}(5.1 \%)$, $\mathrm{Val}_{n-1} \mathrm{COOH}^{+}(2.1 \%), \quad\left[\mathrm{Val}_{n}-\mathrm{H}\right]^{+}(1.2 \%)$ and $\left[\mathrm{Val}_{n^{-}}\right.$ $\left.\mathrm{H}_{2} \mathrm{O}\right]^{+}(0.9 \%)$. The percentage given in parentheses is a measure of the relative contribution of these product ions to the total cation yield and averaged over the cluster sizes from $n=1$ to 30 . The protonated cluster ions $\mathrm{Val}_{n-1} \mathrm{H}^{+}$ dominate the mass spectrum. For $n$ between 4 and 12 this product ion has a relative abundance of $>90 \%$. This value decreases gradually to below $40 \%$ for $n=30$. The yield of the parent cations $\mathrm{Val}_{n}^{+}$increases from about $1 \%$ of the neighboring peak $\mathrm{Val}_{n} \mathrm{H}^{+}$at $n=1$ to more than $20 \%$ at $n=30$. A similar trend was observed previously for methanol and methane cluster ions $[35,36]$.

The yield of parent anions $\mathrm{Val}_{n}^{-}$increases from less than $10 \%$ of $\left[\mathrm{Val}_{n}-\mathrm{H}\right]^{-}$for small $n$ to the same intensity at around $n=30$. The most abundant fragments are: $\left[\mathrm{Val}_{n^{-}}\right.$ $\mathrm{OH}]^{-}(8.5 \%),\left[\mathrm{Val}_{n}-\mathrm{CH}_{3}\right]^{-}(2.5 \%),\left[\mathrm{Val}_{n}-\mathrm{NH}_{2}\right]^{-}(2.5 \%)$, $\left[\mathrm{Val}_{n}-\mathrm{H}_{2} \mathrm{O}\right]^{-}(4.1 \%),\left[\mathrm{Val}_{n}-\mathrm{COOH}\right]^{-}(4.7 \%), \mathrm{Val}_{n-1} \mathrm{H}^{-}$ (5.3\%), $\mathrm{Val}_{n-1} \mathrm{C}_{3} \mathrm{H}_{7}^{-}(2.9 \%)$, and $\mathrm{Val}_{n-1} \mathrm{COOH}^{-}(5.8 \%)$. The percentage given in parentheses is a measure of the relative contribution of these product ions to the total anion yield and averaged over all cluster sizes from $n=1$ to 30 . The relative contribution of the fragment anions increases with the cluster size from $18 \%$ for $n=2$ to $75 \%$ for $n=30$. This trend can also be seen by the symbols plotted in Figure 1: the circles represent the maximum ion yield of the parent or dehydrogenated parent anion whereas the triangles represents the maximum anion yield of $\left[\mathrm{Val}_{n}-\mathrm{OH}\right]^{-}$, which is the dominant fragment for $n>3$.

\subsection{Comparisons with electron ionization of gas phase valine molecules}

Figure 2 shows a portion of a mass spectrum for the cations formed in valine-doped HNDs at an electron energy of $94 \mathrm{eV}$. The present data (solid line) are compared with the mass spectrum for electron ionization of gas phase valine (bar diagram) taken from reference [37]. The two spectra were normalized at the most intense fragment at $m / z=72\left([\text { Val-COOH }]^{+}\right)$.

Very low intensity $\mathrm{ValH}^{+}$is observed in the gas phase (indicating the presence of dimers or clusters in the vapor emitted from heated samples), but it is more than an

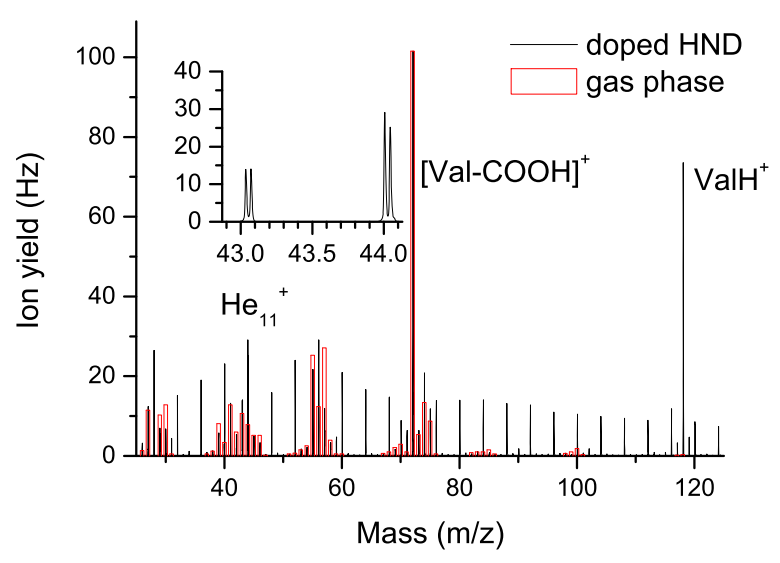

Fig. 2. Section of the mass spectrum obtained for cations formed upon $94 \mathrm{eV}$ electron collisions with valine doped HNDs. Experimental conditions: pressure and temperature of the $\mathrm{He}$ before expansion: $2.3 \mathrm{MPa}$ and $9.75 \mathrm{~K} \rightarrow$ average droplet size $1.5 \times 10^{5}$, temperature of the valine oven: $340 \mathrm{~K}$, electron current $64 \mu \mathrm{A}$. The bar graph represents the mass spectrum obtained by electron ionization of gas phase valine with data taken from the NIST database (at an electron energy of $70 \mathrm{eV}$ ) [37].

order of magnitude more intense from the doped He nanodroplets than the gas phase which agrees well with previous observations from reference [38]. The present mass spectrum agrees also very well with the results obtained by Denifl et al. [20] with a sector field mass spectrometer and HNDs with an average size ten times smaller. $\mathrm{He}_{n}^{+}$ions are responsible for the relatively intense series of peaks with a spacing of $m / z=4$. The insert in Figure 2 shows a small mass range that includes $\mathrm{He}_{11}^{+}$at $m / z=44$. The mass resolution is sufficiently high to separate $\mathrm{He}_{11}^{+}$ from the fragment $\mathrm{CO}_{2}^{+}$that is slightly lower in mass due to a higher nuclear binding of $\mathrm{C}$ and $\mathrm{O}$ compared to He.

In Figure 3 we compare the mass spectra for anion formation from valine doped He nanodroplets at $2 \mathrm{eV}$ (electron bubble) and $22 \mathrm{eV}\left(\mathrm{He}^{*-}\right)$ electronic energy with a mass spectrum that was generated upon summation of ten mass spectra measured at different electron energies via free electron attachment to gas phase valine (taken from Denifl et al. [14]). It is interesting to note that, for both electron energies, the anions from doped He nanodroplets exhibit a higher relative yield of low-mass fragments compared to the gas-phase measurements, i.e., $73 \%(2 \mathrm{eV})$ and $60 \%(22 \mathrm{eV})$ compared to $7 \%$ in the gas phase. In contrast, no fragment ions were observed with He nanodroplets doped with the DNA base thymine [39,40], except for the dehydrogenated parent anion. With the explosive TNT we saw only the parent anion [41]. We explain these observations in terms of the ability of the superfluid He matrix to more efficiently quench the relatively time-consuming fragmentation of the cyclic molecules (in which ring cleavage also occurs) compared to single bond cleavage of a linear molecule. Perhaps the helium matrix also influences intramolecular electron transfer that may accompany the dissociation. 


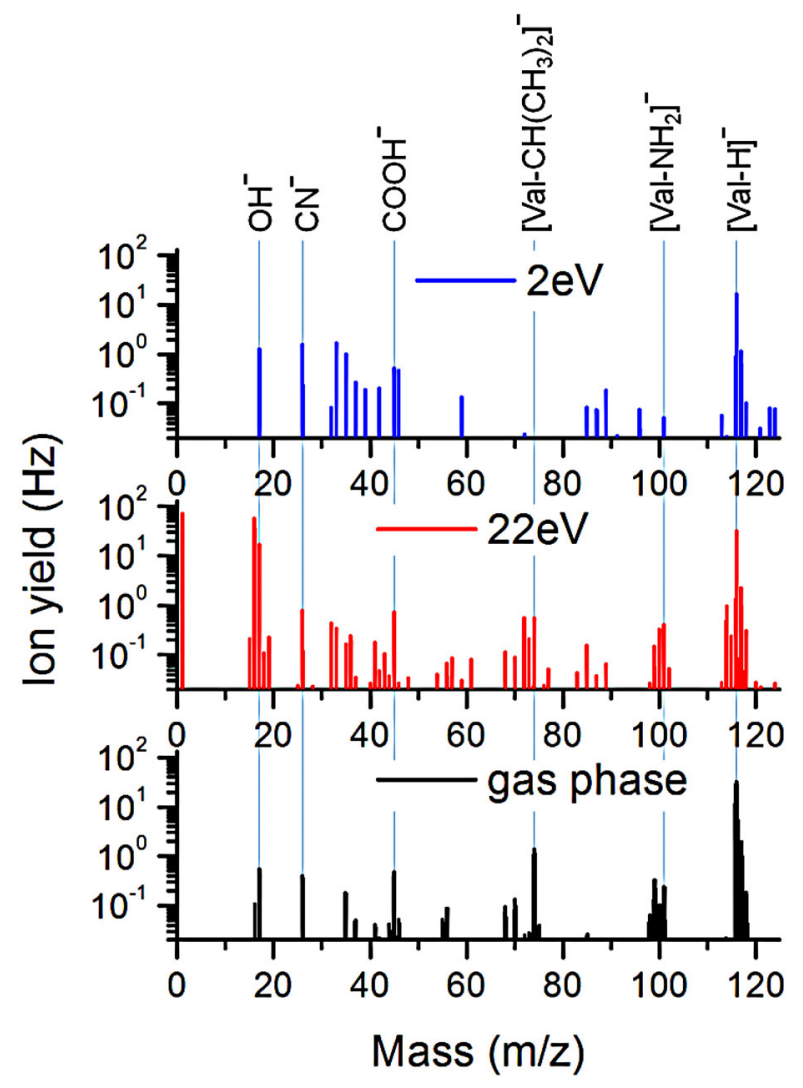

Fig. 3. Mass spectra of negatively charged product ions formed via inelastic electron interactions at $2 \mathrm{eV}$ (upper panel) and $22 \mathrm{eV}$ (middle panel) with He nanodroplets doped with valine compared with free electron attachment to gas phase valine summed up from individual mass spectra measured at several electron energies (see text, data taken from Ref. [14]).

\subsection{Cation formation}

The ionization process in doped He nanodroplets differs strongly from that for electron ionization of gas phase particles. In the nanodroplets, the incoming electron interacts first with one of the He atoms that are in the present case several 10000 times more abundant than the dopant molecules. At electron energies above $19.82 \mathrm{eV}$ electronically excited He atoms can be formed which can induce Penning ionization upon collision with the dopant. Furthermore, the inelastically scattered electron may recombine with the metastable He atom and lead to the formation of $\mathrm{He}^{*-}$ as recently demonstrated [42]. $\mathrm{He}^{*}$ is a heliophobic species and thus Penning ionization of heliophilic dopants deep inside a large helium droplet is unlikely. In contrast, the heliophilic $\mathrm{He}^{*-}$ is highly mobile and efficiently interacts also with heliophilic dopants. The electronic energy of $\mathrm{He}^{*-}(19.74 \mathrm{eV})$ is only slightly lower than that of $\mathrm{He}^{*}[43]$ and thus all dopants, except Ne, can become positively charged via reaction (1).

$$
\mathrm{X}+\mathrm{He}^{*-} \rightarrow \mathrm{X}^{+}+\mathrm{He}+2 \mathrm{e}^{-} .
$$

Figure 4 shows ion efficiency curves for several selected cations formed upon electron bombardment of valine

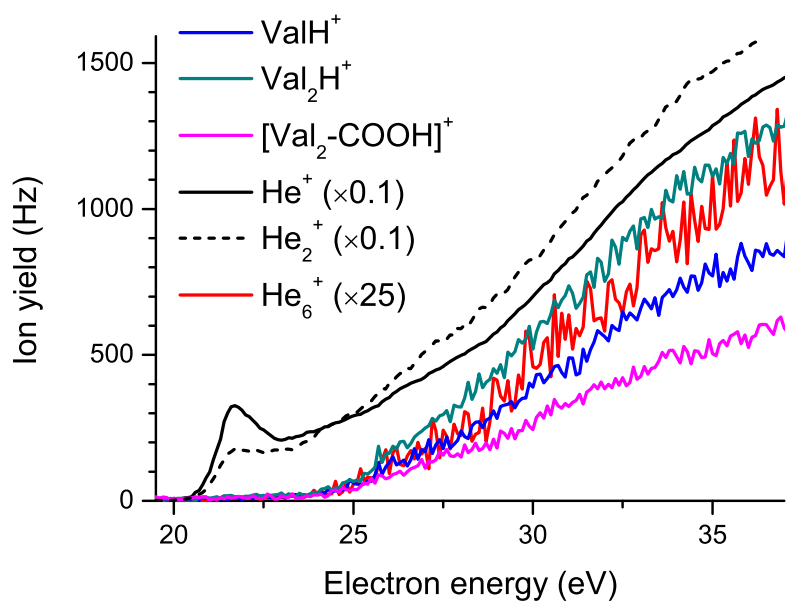

Fig. 4. Ion efficiency curves for selected cations formed via inelastic collisions of electrons with HNDs doped with valine. Temperature and pressure of the He before expansion: $9.7 \mathrm{~K}$, $2.3 \mathrm{MPa}$, electron current: $25 \mu \mathrm{A}$, temperature of the valine oven: $340 \mathrm{~K}$.

doped HNDs. All cationic product ions resulting from valine exhibit a clear threshold at $24.59 \mathrm{eV}$ which is the ionization energy of He. In contrast $\mathrm{He}^{+}$and $\mathrm{He}_{2}^{+}$are additionally formed at lower electron energies via a resonant processes that involves the formation of $\mathrm{He}^{*-}$ [44]. As $\mathrm{He}^{+}$and $\mathrm{He}_{2}^{+}$when formed below the ionization energy of He do not contribute to cation formation of the dopant species inside the HNDs, we conclude that intermediately formed $\mathrm{He}^{*-}$ reacts with a metastable $\mathrm{He}^{*}$ at the surface of the droplet and both possible product ions of this very exothermic reaction, $\mathrm{He}^{+}$and $\mathrm{He}_{2}^{+}$, are ejected from the droplet. This also explains why $\mathrm{He}_{n}^{+}$cluster ions with $n>2$ are not formed via He*-. Furthermore, for the present droplet size of $>10^{5}$, Penning ionization is not contributing to the formation of cations of heliophilic dopants (such as valine). Furthermore, the energy provided by $\mathrm{He}^{+}$ and $\mathrm{He}^{*-}$ may be too high to be quenched by the surrounding He and in many cases is high enough to doubly ionize a dopant cluster. Subsequent Coulomb explosions would then form singly-charged ions as well.

The formation of protonated valine and its clusters appears to dominate cation formation in Figure 1. We attribute the formation of these cations to the dissociative ionization reaction with $\mathrm{He}^{+}$, reaction (2) in accordance with their onset shown in Figure 4 at the ionization energy of He.

$$
\begin{aligned}
\mathrm{He}^{+}+(\mathrm{Val})_{n} \rightarrow & \left((\mathrm{Val})_{n}^{+}\right)^{*} \rightarrow(\mathrm{Val})_{m-1} \mathrm{H}^{+} \\
& + \text {neutral products. }
\end{aligned}
$$

Here the ionization of the valine cluster is accompanied by the dissociation of one (or several) valine molecule(s) and the elimination of $\mathrm{C}_{5} \mathrm{H}_{10} \mathrm{NO}_{2}$ products. The other cations observed in the mass spectrum in Figure 1 are expected to arise in a similar fashion. $\left[\mathrm{Val}_{n}-\mathrm{COOH}\right]^{+}(5.1 \%)$, $\left[\mathrm{Val}_{n}-\left(\mathrm{CH}_{3}\right)_{2} \mathrm{CHCH}\left(\mathrm{NH}_{2}\right)\right]$ or $(\mathrm{Val})_{n-1} \mathrm{COOH}^{+}(2.1 \%)$, $\left[\mathrm{Val}_{n}-\mathrm{H}\right]^{+}(1.2 \%)$ and $\left[\mathrm{Val}_{n}-\mathrm{H}_{2} \mathrm{O}\right]^{+}(0.9 \%)$. Here the neutral fragment is indicated explicitly in the designation of 


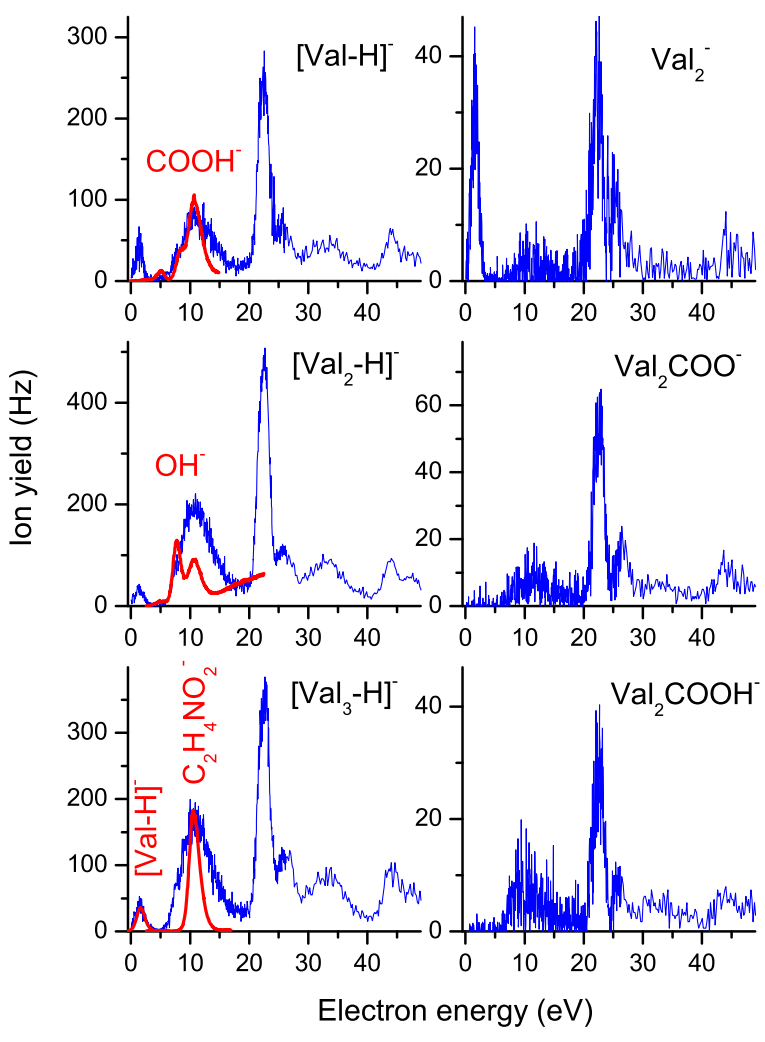

Fig. 5. Anion efficiency curves for some typical product anions formed upon electron attachment to valine clusters embedded in HNDs. $T_{\mathrm{He}}=9.75 \mathrm{~K}, p_{\mathrm{He}}=2.3 \mathrm{MPa}, i_{\mathrm{el}}=64 \mu \mathrm{A}, T_{\mathrm{Val}}=$ $340 \mathrm{~K}$. The solid red lines are fragment anions formed upon dissociative electron attachment to gas phase valine [14] and shifted by $2 \mathrm{eV}$ (see text).

the composition of the cation. The increasing proportion of the parent cation cluster $(\mathrm{Val})_{n}^{+}$as $n$ increases in Figure 1 is simply a reflection of the increasing number of degrees of freedom that can moderate the dissociation process.

\subsection{Anion formation}

The anion efficiency curves of the dehydrogenated parent anions $\left[\mathrm{Val}_{n}-\mathrm{H}\right]^{-}$for $n=1,2$ and 3 (left column) are plotted in Figure 5 together with three abundant product anions in the mass range between the dimer and trimer cluster (right column). The solid red lines represent anion efficiency curves obtained via free electron attachment to gas phase valine, taken from reference [14]. We subtracted the contribution of the isotopomer of $\left[\mathrm{Val}_{n}-\mathrm{H}\right]^{-}$ containing one ${ }^{13} \mathrm{C}$ at $\mathrm{m} / z=117$ from the measured anion efficiency curve. As mentioned in earlier studies, the position resonances of anions formed inside HNDs are shifted by about $2 \mathrm{eV}$, mostly due to the conduction band of He $[39,45,46]$. The parent anions $\mathrm{Val}_{n}^{-}$show two pronounced features at $2 \mathrm{eV}$ and $22 \mathrm{eV}$ as earlier observed for parent anions of DNA bases [39] and other dopants [47-50]. It is interesting to note that for glycine, alanine and serine the intensity of the parent anions is comparable and sometimes larger than the intensity of the corresponding dehydrogenated cluster anion [50]. This may be explained by the substantially larger HNDs utilized in the present study $\left(1.5 \times 10^{5}\right.$ compared to $\left.1.3 \times 10^{4}\right)$. In reference [50] parent cluster anions of amino acids are followed by a series of peaks having a mass separation of four mass units and thus are assigned as $\mathrm{He}_{m} \mathrm{AA}_{n}^{-}$. This suggests that also parent cluster anions of valine, when formed at $2 \mathrm{eV}$, are likely to reside inside the HNDs. The large neutral HNDs chosen in the present study will result in a much larger number of attached He atoms. These anionic complexes will not be accessible for the time of flight mass analysis when they are too heavy to reach the detector within the time delay between two extraction pulses. This also explains the very weak contribution of anions complexed with only a few He atoms. However, similar to the other amino acids picked up by small HNDs [50], we only see these $\mathrm{He}_{m} \mathrm{AA}_{n}^{-}$anions for $n>1$.

Anionic parent cluster ions can emerge only from very few small HNDs of the neutral log-normal distribution. All fragment anions that lose more than one hydrogen atom exhibit just one intense resonance at $22 \mathrm{eV}$ with a weaker feature at $25-26 \mathrm{eV}$. This indicates that the superfluid He matrix is efficiently quenching more complex and relatively slow fragmentation reactions [40] and we propose that most low-mass fragments of amino acids are formed via an intermediate $[\mathrm{AA}-\mathrm{H}]^{*-}$ as hydrogen loss is the most efficient DEA channel to compete with autodetachment. Instead of the low-mass fragments observed via DEA to gas phase amino acid molecules, these channels contribute to the dehydrogenated parent anions $\left[\mathrm{Val}_{n}-\mathrm{H}\right]^{-}$ when formed inside HNDs.

The three most abundant fragments $\mathrm{OH}^{-}, \mathrm{COOH}^{-}$, and $\mathrm{C}_{2} \mathrm{H}_{4} \mathrm{NO}_{2}^{-}$(where most likely the aliphatic side chain $\mathrm{H}_{3} \mathrm{C}-\mathrm{CH}-\mathrm{CH}_{3}$ is lost) from reference [14] are shown as solid red lines and the energy axis is shifted by about $2 \mathrm{eV}$ to compensate for the energy an electron requires to enter the conduction band of liquid He [45]. The resonances of these fragment anions fit very well with the anion efficiency curves of $\left[\mathrm{Val}_{n}-\mathrm{H}\right]^{-}$in the corresponding electron energy range. This points to a matrix effect in the superfluid He which stabilizes an intermediately formed $\left[\mathrm{Val}_{n}\right.$ $\mathrm{H}]^{*-}$ that, when isolated, is prone to further dissociation in the fs to $\mu$ s time regime.

As observed in earlier studies, we observe a repetition of the resonances after $22 \mathrm{eV}$ which can be explained by an inelastic scattering event of an electron at He, forming $\mathrm{He}^{*}$ (which requires an energy of $19.82 \mathrm{eV}$ for the formation of $\mathrm{He}^{*}$ in the $2^{3} \mathrm{~S}_{1}$ state) [39,47-51]. It is interesting to note that at $22 \mathrm{eV}$ all anions are formed, including those that cannot be formed via low-energy electron attachment close to $0 \mathrm{eV}$. Whereas for small HNDs (average size $<10^{4}$ ) inelastically scattered electrons will be attracted by neighboring dopant clusters, for large HNDs (average size $>10^{5}$, as in the present study) the formation of an intermediate $\mathrm{He}^{*-}$ becomes more likely which finally will interact with the dopant cluster. As already mentioned in Section 3.1, the relative yield of non-stoichiometric valine cluster anions (sum of the yield of all fragment anions divided by 


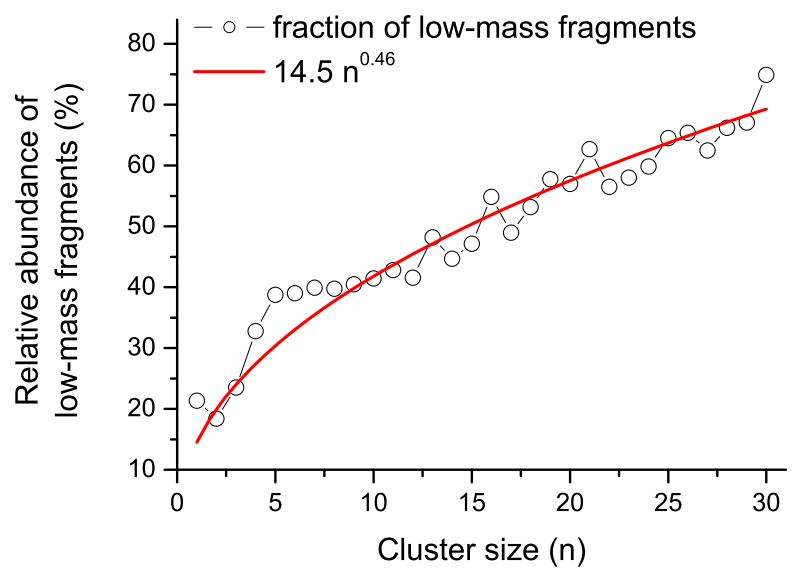

Fig. 6. The relative abundance of the total low-mass fragment anion yield (all anions without $\mathrm{Val}_{n}^{-}$and $\left[\mathrm{Val}_{n}-\mathrm{H}\right]^{-}$divided by the total anion yield for a given cluster size $n$ ) is plotted as a function of $n$ (open circles). The data are deduced from the mass spectrum shown in Figure 1. The red solid line is the fit of a power function to the data points.

the anion yield of the parent and dehydrogenated parent anion for a given cluster size) increases monotonically from $18 \%$ for $n=2$ to $75 \%$ for $n=30$.

The capture cross section of a HND $\mathrm{He}_{\mathrm{N}}$ is basically the geometric cross section and thus proportional to $N^{2 / 3}$. In order to pick up $n$ dopant molecules the cross sections of the HND has to be $n$ times larger than for a HND that will pick up only one dopant. Thus the number of He atoms required for picking up $n$ molecules has to be $n^{1.5}$ times larger than for a HND picking up one molecule. The loss of He atoms due to the pickup of individual valine molecules and the release of binding energy upon dopant cluster formation scales with $n$, the number of dopants being picked up. For large HNDs the loss of He due to the pickup process and the release of binding energy upon dopant cluster formation can be neglected as it scales only with $N^{2 / 3}$. The radius of the HND scales with $N^{1 / 3}$ and thus the radius of the initial droplet scales with the square root of the number of dopants $n$ that are picked up $\left(n^{1 / 2}\right)$. In Figure 6 the fraction of low-mass fragments is plotted as a function of the cluster size (open symbols) and compared with a power function (solid line). The exponent is very close to $1 / 2$ which is a measure for the radius of the HND. The radius of a HND containing $10^{5} \mathrm{He}$ atoms is with about $11 \mathrm{~nm}$ much larger than size of a valine molecule. Thus the radius of the HND is a good approximation for the thickness of the He layer surrounding the dopant cluster. So larger dopant clusters are surrounded by a thicker layer of He. As a consequence $\mathrm{He}^{*-}$ becomes more important for the anion formation. Therefore, the fragmentation of dopant molecules increases by the transfer of the excitation energy of $19.74 \mathrm{eV}$ from $\mathrm{He}^{*-}$ to the valine cluster. If fragmentation is mainly due to $\mathrm{He}^{*-}$, we conclude that the importance of $\mathrm{He}^{*-}$ is proportional to the thickness of the He layer covering the dopant.

The anion efficiency curves in the right panels of Figure 5 show that fragment anions are predominantly formed

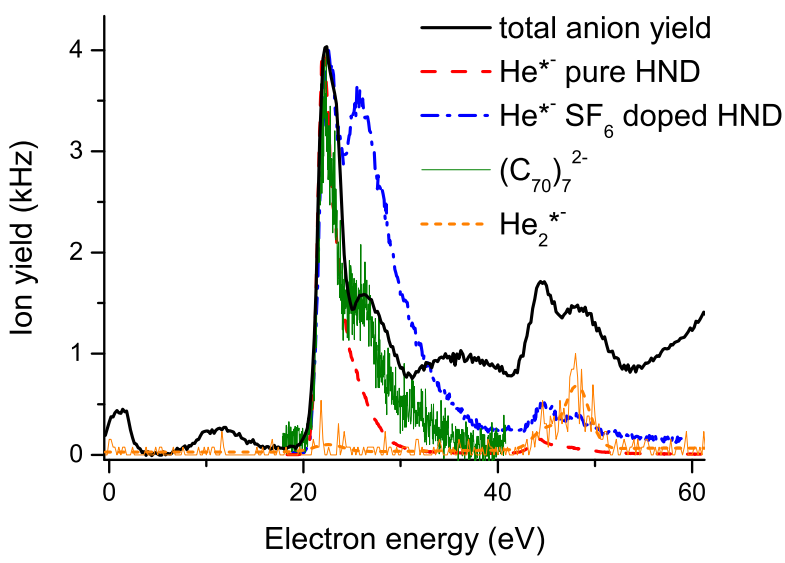

Fig. 7. Ion efficiency curve of the total anion signal measured from valine doped HNDs, integrated from $m / z=1$ up to $m / z=1200$ (solid black line). Parameters: $T_{\mathrm{He}}=9.75 \mathrm{~K}$, $p_{\mathrm{He}}=2.3 \mathrm{MPa}, i_{\mathrm{el}}=64 \mu \mathrm{A}, T_{\mathrm{Val}}=340 \mathrm{~K}$. The $\mathrm{He}_{2}{ }^{*-}$ was taken from the same measurement. The data for $\mathrm{He}^{*-}$ from pristine and $\mathrm{SF}_{6}$ doped HNDs were taken from [42,52] and for $\left(\mathrm{C}_{70}\right)_{7}^{2-}$ from reference [53].

at the $22 \mathrm{eV}$ resonance that we assign to the formation of an intermediate $\mathrm{He}^{*-}[42,43]$. In Figure 7 the total anion yield (solid black line) is plotted in comparison to the anion yield of $\mathrm{He}^{*-}$ from pristine (red dashed line, data taken from [42] and $\mathrm{SF}_{6}$ doped (blue dash dotted line, data taken from [52]) HNDs and the anion efficiency curve of $\left(\mathrm{C}_{70}\right)_{7}^{2-}$ (thin green line, data taken from [53]). The importance of $\mathrm{He}^{*-}$ in the formation of anions from doped HNDs with an average size larger than $10^{5}$ He atoms becomes very obvious from the total anion yield. Both, at $22 \mathrm{eV}$ and $44 \mathrm{eV}$ a pronounced maximum can be seen, followed by a slightly less intense and wider peak centered at $26 \mathrm{eV}$ and $48 \mathrm{eV}$, respectively. Although $\mathrm{He}^{*-}$ is strongly affected by the presence of dopants such as $\mathrm{SF}_{6}$ (red, dashed line: undoped HNDs, blue, dash-dotted line: $\mathrm{SF}_{6}$ doped HNDs, data taken from $\left.[42,52]\right), \mathrm{He}_{2}{ }^{*-}$ is relatively unaffected $[42,52]$. In reference [52] we interpreted the peak at $26 \mathrm{eV}$ as a $\mathrm{He}^{*-}$ in a higher excited state that in gas phase quickly decays but in the HND is stabilized. The black curve even exhibits a clear shoulder at $23 \mathrm{eV}$ that could be assigned to $\mathrm{He}^{*-}\left(1 s 2 p^{2}\right)$ which is $1 \mathrm{eV}$ higher in energy than $\mathrm{He}^{*-}$ in the $1 s 2 s 2 p$ state. In contrast to reference [42], in the present study also $\mathrm{He}_{2}{ }^{*-}$ (orange lines, the dashed orange line is a fit to the measurement to guide the eye) is barely visible at $23 \mathrm{eV}$ but clearly present at $45 \mathrm{eV}$ and $48 \mathrm{eV}$, where each electron is able to generate two metastable He atoms.

\section{Conclusions}

Formation of anions and cations via inelastic electron interactions with valine doped HNDs was investigated with high-resolution mass spectrometry. In contrast to an earlier study where only cations from ten times smaller HNDs doped with valine [20] and clusters $\mathrm{Val}_{n} \mathrm{H}^{+}$up to $n=8$ 
were measured, we utilized ten times larger HNDs and measured clusters up to $n>40$. The negatively charged product ions exhibit a pronounced increase of fragmentation with the size of the HNDs which can be attributed to the enhanced formation of metastable helium anions $\mathrm{He}^{*-}$. Even in a single measurement this size effect can be observed as small dopant clusters are preferentially formed by pickup of small HNDs and large dopant clusters by pickup of large HNDs. Cation efficiency curves were measured for the complete mass spectrum and, as expected, product ions from valine clusters embedded in HNDs are exclusively formed via electron transfer to an initially formed $\mathrm{He}^{+}$at electron energies higher than $24.59 \mathrm{eV}$. This confirms that valine is a heliophilic dopant and moves around the center of a HND [54] where it most likely interacts with $\mathrm{He}^{+}$or $\mathrm{He}^{*-}$. Anions from HNDs doped with valine were measured for the first time and significant differences to earlier measurements of HNDs doped with other amino acids were identified. The resonance at $22 \mathrm{eV}$ is by far the most efficient energy for anion formation which is a result of the ten times larger average HNDs. Anion formation at low electron energies is strongly suppressed for large HNDs in comparison to smaller droplets $[39,47,50,51]$. We explain this (i) by the low mobility of the electron bubble [43,55-57] and (ii) by the reduced ejection probability of parent cluster anions of amino acids in case they are formed. Also, in the energy range of DEA that is typically assigned to core excited resonances, the fragmentation pattern differs strongly between doped HNDs and the gas phase. In HNDs the dominant product is the close-shell dehydrogenated parent anion $\left[\mathrm{Val}_{n}-\mathrm{H}\right]^{-}$, whereas low-mass fragments are dominant for DEA to gas phase molecules. We attribute this difference to a matrix effect in the superfluid He which stabilizes an intermediately formed $\left[\mathrm{Val}_{n}-\mathrm{H}\right]^{*-}$ that, when isolated, is prone to further dissociation in the fs to $\mu$ s time regime. However, the encounter of a dopant valine cluster with $\mathrm{He}^{*-}$ provides $19.7 \mathrm{eV}$ of electronic energy in addition to the extra electron, which explains the enhanced anion fragmentation compared to electron attachment. This also explains why anions that cannot be formed via electron attachment at low electron energies, such as $\mathrm{COOH}^{-}$, exhibit a strong resonance at $22 \mathrm{eV}$ with doped HNDs. An inelastically scattered electron cannot form these anions, however, $\mathrm{He}^{*-}$ clearly can. In the present study the effect of the HND on valine anion formation is demonstrated for the first time: the larger a HND, the more important becomes $\mathrm{He}^{*-}$. The electronic energy of this metastable species strongly enhances dissociation of molecular constituents of the dopant cluster. In addition, we deduce a linear dependence between the ionization efficiency with $\mathrm{He}^{*-}$ and the radius of the HND which is a measure for the thickness of the He layer covering the dopant.

This work was supported by the Austrian Science Fund, FWF (P26635, I978, P24443). This work is dedicated to Michael Alan, a very gifted experimentalist in the field of the interaction of low-energy electrons with molecules. He achieved incredibly high energy-resolution for his free electron beams and obtained thereby enormous insight into these fundamental collision processes. He is a true gentleman and we will miss his contribution to conferences and the literature.

\section{References}

1. M.A. Śmiałek, P. Limão-Vieira, N.J. Mason, A.V. Solov'yov, Eur. Phys. J. D 68, 312 (2014)

2. E. Alizadeh, T.M. Orlando, L. Sanche, Ann. Rev. Phys. Chem. 66, 379 (2015)

3. I. Baccarelli, I. Bald, E. Illenberger, F.A. Gianturco, J. Kopyra, Phys. Rep. 508, 1 (2011)

4. R. Janečková, D. Kubala, O. May, J. Fedor, M. Allan, Phys. Rev. Lett. 111, 213201 (2013)

5. M. Allan, Phys. Rev. Lett. 98, 123201 (2007)

6. B.C. Ibănescu, O. May, M. Allan, Phys. Chem. Chem. Phys. 10, 1507 (2008)

7. Y. Hu, E.R. Bernstein, J. Chem. Phys. 128, 164311 (2008)

8. A.F. Lago, L.H. Coutinho, R.R.T. Marinho, A. Naves de Brito, G.G.B. de Souza, Chem. Phys. 307, 9 (2004)

9. P. Papp, J. Urban, Š. Matejčík, M. Stano, O. Ingolfsson, J. Chem. Phys. 125, 204301 (2006)

10. P. Papp, P. Shchukin, J. Kočíšek, Š. Matejčík, J. Chem. Phys. 137, 105101 (2012)

11. H.-W. Jochims, M. Schwell, J.-L. Chotin, M. Clemino, F. Dulieu, H. Baumgärtel, S. Leach, Chem. Phys. 298, 279 (2004)

12. V. Vorsa, T. Kono, K.F. Willey, N. Winograd, J. Phys. Chem. B 103, 7889 (1999)

13. S. Maclot, M. Capron, R. Maisonny, A. Lawicki, A. Méry, J. Rangama, J.-Y. Chesnel, S. Bari, R. Hoekstra, T. Schlathölter, B. Manil, L. Adoui, P. Rousseau, B.A. Huber, ChemPhysChem 12, 930 (2011)

14. S. Denifl, H.D. Flosadóttir, A. Edtbauer, O. Ingólfsson, T.D. Märk, P. Scheier, Eur. Phys. J. D 60, 37 (2010)

15. H. Dögg Flosadottir, S. Denifl, F. Zappa, N. Wendt, A. Mauracher, A. Bacher, H. Jonsson, T.D. Märk, P. Scheier, O. Ingolfsson, Angew. Chem. Int. Ed. 46, 8057 (2007)

16. T. Schlathölter, F. Alvarado, S. Bari, A. Lecointre, R. Hoekstra, V. Bernigaud, B. Manil, J. Rangama, B. Huber, ChemPhysChem 7, 2339 (2006)

17. M. Neustetter, J. Aysina, F.F. da Silva, S. Denifl, Angew. Chem. Int. Ed. 54, 9124 (2015)

18. J.-C. Poully, V. Vizcaino, L. Schwob, R. Delaunay, J. Kocisek, S. Eden, J.-Y. Chesnel, A. Mery, J. Rangama, L. Adoui, B. Huber, ChemPhysChem 16, 2389 (2015)

19. J.P. Toennies, A.F. Vilesov, Angew. Chem., Int. Ed. 43, 2622 (2004)

20. S. Denifl, I. Mähr, F. Ferreira da Silva, F. Zappa, T.D. Märk, P. Scheier, Eur. Phys. J. D 51, 73 (2009)

21. B. Bellina, D.J. Merthe, V.V. Kresin, J. Chem. Phys. 142, 114306 (2015)

22. Y. Ren, V.V. Kresin, J. Chem. Phys. 128, 074303 (2008)

23. Y. Ren, R. Moro, V.V. Kresin, Eur. Phys. J. D 43, 109 (2007)

24. X. Zhang, N.B. Brauer, G. Berden, A.M. Rijs, M. Drabbels, J. Chem. Phys. 136, 044305 (2012)

25. N.B. Brauer, S. Smolarek, X. Zhang, W.J. Buma, M. Drabbels, J. Phys. Chem. Lett. 2, 1563 (2011)

26. W. Kong, L. Pei, J. Zhang, Int. Rev. Phys. Chem. 28, 33 (2009) 
27. F. Bierau, P. Kupser, G. Meijer, G. von Helden, Phys. Rev. Lett. 105, 133402 (2010)

28. U. Henne, J.P. Toennies, J. Chem. Phys. 108, 9327 (1998)

29. R. Sliter, L.F. Gomez, J. Kwok, A. Vilesov, Chem. Phys. Lett. 600, 29 (2014)

30. L.F. Gomez, E. Loginov, R. Sliter, A.F. Vilesov, J. Chem. Phys. 135, 154201 (2011)

31. L. An der Lan, P. Bartl, C. Leidlmair, H. Schoöbel, R. Jochum, S. Denifl, T.D. Maärk, A.M. Ellis, P. Scheier, J. Chem. Phys. 135, 044309 (2011)

32. H. Schöbel, C. Leidlmair, P. Bartl, A. Aleem, M. Hager, O. Echt, T.D. Märk, P. Scheier, Phys. Chem. Chem. Phys. 13, 1092 (2011)

33. S. Ralser, J. Postler, M. Harnisch, A.M. Ellis, P. Scheier, Int. J. Mass Spectrom. 379, 194 (2015)

34. O. Echt, K. Sattler, E. Recknagel, Phys. Rev. Lett. 47, $1121(1981)$

35. M. Goulart, P. Bartl, A. Mauracher, F. Zappa, A.M. Ellis, P. Scheier, Phys. Chem. Chem. Phys. 15, 3577 (2013)

36. C. Leidlmair, P. Bartl, H. Schöbel, S. Denifl, S. Yang, A.M. Ellis, P. Scheier, ChemPhysChem 13, 469 (2012)

37. S.E. Stein, NIST Mass Spec Data Center, NIST Chemistry Webbook, NIST Standard Reference Database Number 69, edited by P.J. Linstrom, W.G. Mallard (National Institute of Standards and Technology, Gaithersburg MD, 20899), http://webbook.nist.gov/chemistry (Access Date December 15th 2015)

38. S.F. Yang, S.M. Brereton, M.D. Wheeler, A.M. Ellis, Phys. Chem. Chem. Phys. 7, 4082 (2005)

39. S. Denifl, F. Zappa, I. Mähr, J. Lecointre, M. Probst, T.D. Märk, P. Scheier, Phys. Rev. Lett. 97, 043201 (2006)

40. S. Denifl, F. Zappa, A. Mauracher, F. Ferreira da Silva, A. Bacher, O. Echt, T.D. Märk, D.K. Böhme, P. Scheier, ChemPhysChem 9, 1387 (2008)

41. A. Mauracher, H. Schöbel, F. Ferreira da Silva, A. Edtbauer, C. Mitterdorfer, S. Denifl, T.D. Märk, E. Illenberger, P. Scheier, Phys. Chem. Chem. Phys. 11, 8240 (2009)

42. A. Mauracher, M. Daxner, J. Postler, S.E. Huber, S. Denifl, P. Scheier, J.P. Toennies, J. Phys. Chem. Lett. 5, 2444 (2014)

43. S.E. Huber, A. Mauracher, J. Phys. Chem. A 118, 6642 (2014)
44. M. Renzler, M. Daxner, N. Weinberger, S. Denifl, P. Scheier, O. Echt, Phys. Chem. Chem. Phys. 16, 22466 (2014)

45. A.L. Fetter, in The Physics of Liquid and Solid Helium, edited by K.H. Bennemann, J.B. Ketterson (Wiley, New York, 1976), Pt. 1, p. 207, and references therein

46. K. Martini, J.P. Toennies, C. Winkler, Chem. Phys. Lett. 178, 429 (1991)

47. J. Postler, V. Vizcaino, S. Denifl, F. Zappa, S. Ralser, M. Daxner, E. Illenberger, P. Scheier, J. Phys. Chem. A 118, 6553 (2014)

48. F. Ferreira da Silva, S. Ptasinska, S. Denifl, D. Gschliesser, J. Postler, C. Matias, T.D. Märk, P. Limão-Vieira, P. Scheier, J. Chem. Phys. 135, 174504 (2011)

49. F. Ferreira da Silva, S. Jaksch, G. Martins, H.M. Dang, M. Dampc, S. Denifl, T.D. Märk, P. Limao-Vieira, J. Liu, S. Yang, A.M. Ellis, P. Scheier, Phys. Chem. Chem. Phys. 11, 11631 (2009)

50. F. Ferreira da Silva, S. Denifl, T.D. Märk, A.M. Ellis, P. Scheier, J. Chem. Phys. 132, 214306 (2010)

51. F. Zappa, S. Denifl, I. Mähr, A. Bacher, O. Echt, T.D. Märk, P. Scheier, J. Am. Chem. Soc. 130, 5573 (2008)

52. A. Mauracher, M. Daxner, S.E. Huber, J. Postler, M. Renzler, S. Denifl, P. Scheier, A.M. Ellis, J. Chem. Phys. 142, 104306 (2015)

53. A. Mauracher, M. Daxner, S.E. Huber, J. Postler, M. Renzler, S. Denifl, P. Scheier, A.M. Ellis, Angew. Chem. Int. Ed. 53, 13794 (2014)

54. A.W. Hauser, A. Volk, P. Thaler, W.E. Ernst, Phys. Chem. Chem. Phys. 17, 10805 (2015)

55. M. Rosenblit, J. Jortner, J. Chem. Phys. 124, 194505 (2005)

56. M. Rosenblit, J. Jortner, J. Chem. Phys. 124, 194506 (2005)

57. W. Wei, Z.L. Xie, G.M. Seidel, H.J. Maris, J. Low Temp. Phys. 171, 178 (2013)

Open Access This is an open access article distributed under the terms of the Creative Commons Attribution License (http://creativecommons.org/licenses/by/4.0), which permits unrestricted use, distribution, and reproduction in any medium, provided the original work is properly cited. 